\section{Rocuronium in paediatric ICU}

To the Editor:

Dr. J.D. Tobias' paper describing rocuronium infusion in the paediatric intensive care setting (Can J Anaesth 1996; 43: 353 -7 ) is a valuable addition to the literature. The PICU is as well populated with "therapeutic orphans" as is paediatric anaesthesia and any attempts to provide a scientific basis for the use of drugs in this setting are to be applauded.

Rocuronium use is currently spreading as many hospital pharmacies withdraw vecuronium from general use. Thus many PICUs may be considering the use of rocuronium as a replacement. This is the first publication to describe the use of rocuronium as an infusion for several days' duration.

However, the results should not be over interpreted. As Dr. Tobias points out, the wide age range included in the study may be one factor in the dose variability described. The conclusion that regular monitoring of neuromuscular blockade in this setting is essential is well taken. However, an age-related dose range would be very useful to clinicians. No relationship between age and dose was described - was there one? Also a graphical breakdown of age would be useful in interpreting the data e.g., how many patients fell within the $<6$ months, $<1$ year etc. ranges?

Other factors affecting dose include concurrent administration of drugs which may affect metabolism or effect of rocuronium. Since some patients were being treated for elevated ICP these may have been receiving barbiturates. If so were liver enzymes mildly induced (Dr. Tobias excludes LFTs of $\geq$ twice normal)? A list of concurrent medications would be helpful.

Until, and almost certainly after, we have more extensive information on dose requirements at different age ranges regular neuromuscular monitoring of these patients should be employed.

D. Withington BM MRCP FRCA

Departments of Anaesthesia \& PICU

McGill University, Montréal, PQ

\section{REPLY}

Thank you for your interest and comments concerning my paper reporting the use of rocuronium for neuromuscular blockade in the paediatric ICU patient.' I share your interest in information concerning the appropriate use of new agents in the paediatric ICU population. Unfortunately, as you have pointed out, paediatric ICU patients may be "therapeutic orphans" in that we have limited clinical information concerning the use of many therapeutic agents in this population as opposed to adult patients. While the adult studies may provide a basis for the use of these agents in the paediatric population, considerable differences can occur in children and we should not think of them as merely small adults.

Unfortunately, because of the limited number of patients in the study $(n=20)$, I am unable to make any definite conclusions concerning differences in dosing requirements in regard to age or concurrent medication use. When analyzed, no differences were noted with regard to age; however, with a sample size of 20 and only four patients $<12$ months, it is possible that a type II error existed. Likewise, there were only two patients receiving barbiturates and therefore no conclusion can be drawn regarding the effects of barbiturates on rocuronium dosing requirements. In a previous study, ${ }^{2}$ we noted an increase in pancuronium requirements $(0.056$ vs 0.14 $\left.m g \cdot \mathrm{kg}^{-1} \cdot \mathrm{hr}^{-1}, P<0.05\right)$ in seven of 25 patients receiving anticonvulsants including barbiturates, phenytoin, and carbamazepine.

Regardless of these issues, I concur that whenever neuromuscular blocking agents are used, monitoring of neuromuscular blockade is strongly recommended. Both of our previous studies, ${ }^{1,2}$ have demonstrated eight- to 10-fold variations in the doses required to maintain one twitch of the train-of-four. Such variability is to be expected in a population as varied as the paediatric ICU population. This variability may relate to age, underlying illness or the administration of other medications. Additionally, while rocuronium offers the advantage of limited cardiovascular effects, as it is dependent on hepatic metabolism, other agents (atracurium or cis-atracurium) may be more appropriate in patients with hepatic dysfunction.

Joseph D. Tobias MD

Pediatric Critical Care/Anesthesia

The University of Missouri

Department of Child Health

M658 Health Sciences Center

One Hospital Drive, Columbia, Missouri 65212

Fax: (573) 882-2742: Tel: (573) 882-6544

\section{REFERENCES}

1 Tobias $J D$. Continuous infusion of rocuronium in a paediatric intensive care unit. Can J Anaesth 1996; 43: 353-7.

2 Tobias JD, Lynch A, McDuffee, Garrett JS. Pancuronium infusion for neuromuscular blockade in children in the pediatric intensive care unit. Anesth Analg 1995; 81: 13-6.

\section{Aseptic meningitis using the needle-through-needle technique}

To the Editor:

Cascio and Heath' described a case report of mẹningitis following a combined spinal-epidural technique in a labouring term parturient. They used a $17 \mathrm{G}$ Hustead needle (Concord/ Portex, Keene, NH 03431) for the epidural and a $25 \mathrm{G} 12.3 \mathrm{~cm}$ long Quincke spinal needle (Becton/Dickinson, Franklin Lakes, NJ 07417) for the spinal by the needle-through-needle technique. Sixteen hours after delivery the parturient's body temperature increased to $38.7^{\circ}$ and she complained of a nonpositional frontal headache. This was associated with chills, photophobia and mild nuchal rigidity. Diagnostic lumbar puncture revealed cloudy CSF with increased polymorphonuclear white cell count, increased protein and decreased glucose concentrations. All cultures from the CSF remained negative until $72 \mathrm{hr}$ when a single broth culture grew a possible contaminant, streptococcus salivarius with no bacterial growth on the culture plates. Despite that, an empirical diagnosis of bacterial meningitis was made and antibiotics were started with vancomycin $1 \mathrm{~g}$ every $12 \mathrm{hr}$ and ceftriaxone $2 \mathrm{~g}$ every $8 \mathrm{hr} i \mathrm{v}$. The patient was afebrile after $12 \mathrm{hr}$ and asymptomatic by 24 hr.

In their discussion, Cascio and Heath described the two cases of Harding et al. ${ }^{2}$ However, they did not mention my comment on that article. ${ }^{3}$

Their case can be a case of aseptic meningitis due to metallic micro particles produced by the needle-through-needle technique for combined spinal-epidural anaesthesia. The details of this proposal were described in several letters. ${ }^{4-6} \mathrm{~A}$ 
recent article ${ }^{7}$ also confirmed the finding of a "notching defect... within the lumen along the tract of the spinal needle at the tip" of the epidural needle in the needle-through-needle technique. This "notching defect" is an accumulation of metallic micro particles detached from its surface, which can be pushed forward by the force exerted to insert the epidural catheter into the epidural space after withdrawing the spinal needle from the lumen of the epidural needle. Cascio and Heath used the Quincke type spinal needle which causes much more friction and metallic micro particles production than the pencil point spinal needles while introducing it through the bent epidural needle's tip. This combination is also not allowed by the FDA.

Joseph Eldor MD

Department of Anaesthesia

Misgav Ladach General Hospital

PO Box 12142

Jerusalem 91120 , Israel

\section{REFERENCES}

1 Cascio $M$, Heath $G$. Meningitis following a combined spinal-epidural technique in a labouring term parturient. Can J Anaesth 1996; 43: 399-402.

2 Harding SA, Collis RE, Morgan BM. Meningitis after combined spinal-extradural anaesthesia in obstetrics. $\mathrm{Br} \mathrm{J}$ Anaesth 1994; 73: 545-7.

3 Eldor J, Guedj P. Aseptic meningitis due to metallic particles in the needle-through-needle technique (Letter). Reg Anesth 1995; 20: 360.

4 Eldor J, Brodsky V. Danger of metallic particles in the spinal-epidural spaces using the needle-through-needle approach (Letter). Acta Anaesthesiol Scand 1991; 35: 461.

5 Eldor J. Metallic particles in the spinal-epidural needle technique (Letter). Reg Anesth 1994; 19: 219-20.

6 Carrie $L E$. Metallic fragments and the combined spinalextradural technique (Letter). Br J Anaesth 1992; 69: 662-3.

7 Herman N, Molin J, Knape KG. No additional metal particle formation using the needle-through-needle combined epidural/spinal technique. Acta Anaesthesiol Scand 1996; 40: 227-31.

\section{REPLY}

Reviewing the patient's course in our case report' it is clear to see this is a case of bacterial meningitis and not aseptic meningitis. The diagnosis was made based on the appearance, cytology and chemistry of the CSF. The CSF was cloudy, had raised protein concentration, lowered glucose concentration. Cultures from the CSF were negative. Failure to isolate a bacterial pathogen does not disprove a bacterial aetiology because gram stain and cultures may be negative in up to $10 \%$ of cases of bacterial meningitis. ${ }^{2}$ The CSF to serum glucose concentration ratio in our patient was 0.36 . Briem found $a$ CSF to serum glucose ratio of $<0.4$ to be specific for the diagnosis of bacterial meningitis. ${ }^{3}$

Harding et al. ${ }^{4}$ reported two cases of meningitis following CSE techniques. The first case was an aseptic meningitis following a combined spinal-epidural technique for labor analgesia. We did not cite Dr. Eldor's comment to the above case report in our report because (1) the report was not a study but a letter, (2) our patient had a clear-cut case of bacterial meningitis and (3) most importantly, we do not believe that metallic microparticles induce aseptic meningitis. There is no evidence that such particles cause any problems if inserted into the epidural space, subarachnoid space or any bodily compartment. We have performed several thousand combinedtechniques for labour analgesia using the needle through needle technique and this is the only case of meningitis of any type we have seen in our institution.

Dr. Eldor is incorrect when he states that the use of a Quinke needle for a needle-through-needle technique is not allowed by the FDA. There are several prepackaged commercial kits available for performing combined technique using the needle-through-needle technique in which the combination of an epidural needle and a Quinke Spinal needle are used. In order for those kits to be sold they must be approved by the FDA (personal communication-Becton Dickinson Co.).

The combined spinal-epidural using the needle-throughneedle technique is a valuable tool for providing regional anesthesia. We should allow an unsubstantiated theory to remove this tool from our arsenal.

\section{Martin Cascio MD}

Department of Anesthesiology

Magee-Womens Hospital

300 Halket Street

Pittsburgh, PA 15213, USA

\section{REFERENCES}

1 Cascio $M$, Heath $G$. Meningitis following a combined spinal-epidural technique in a labouring term parturient. Can J Anaesth 1996; 43: 399-402.

2 Feigin $R D$, Shackelford $P G$. Value of repeat lumbar puncture in the differential diagnosis of meningitis. $\mathrm{N}$ Engl $\mathbf{J}$ Med 1976; 289: 571-4.

3 Briem $\mathrm{H}$. Comparison between cerebrospinal fluid concentrations of glucose, total protein, chloride, lactate, and total amino acids for the differential diagnosis of patients with meningitis. Scand J Infect Dis 1983; 15: 277-84.

4 Harding SA, Collis RE, Morgan BM. Meningitis after combined spinal-extradural anaesthsia in obstetrics. $\mathrm{Br} J$ Anaesth 1994; 73: 545-7.

5 Eldor J, Guedj P. Aseptic meningitis due to metallic particles in the needle-through-needle technique (Letter). Reg Anesth 1995; 20: 360.

\section{Erratum}

Boushra NN. Anaesthetic management of patients with sleep apnoea syndrome. Can J Anaesth 1996; 43: 599-616.

Please note the typographic errors as follows:

Page 610, right column, line 2: " $\geq 45 \mathrm{~cm} \mathrm{H}_{2} \mathrm{O}$ " should read " $>-45 \mathrm{~cm} \mathrm{H}_{2} \mathrm{O}$ ". i.e.,

ry pressure of $>-45 \mathrm{~cm} \mathrm{H}_{2} \mathrm{O}$ and sustained head raising

Page 612, 2nd paragraph, line 2: "Microlaryngoscopy" should read "Midline glossectomy", i.e.,

the base of the tongue. Midline glossectomy and lin- 